\title{
Simulation of Tool Vibration Control in Turning, Using a Self-Sensing Actuator
}

\author{
B. H. FREYER \\ N. J. THERON \\ P. S. HEYNS \\ Dynamic Systems Group, Department of Mechanical and Aeronautical Engineering, University \\ of Pretoria, Pretoria 0002, South Africa (bhfreyer@tuks.co.za)
}

\begin{abstract}
Besides reducing the restricting effects of tool vibrations on productivity, work-piece surface finish and tool life, it is desirable to handle lack of space for sensors at the tool tip and the cost of control systems in turning processes in an effective way. This work considers these two aspects by exploiting the concept of a self-sensing actuator (SSA) in the simulation of tool vibration control. The tool holder structure, in its passive as well as active state, is modeled as a supported cantilever. A feedback filtered-x least-mean-square (LMS) algorithm is chosen to compute the control action. A known technique, which consists of pre-filtering the inputs to the LMS-algorithm maintains the stability of the control system. The self-sensing path is modeled and illustrated. It consists of the transmission of the tool tip displacement to the SSA where it is sensed by converting it into a voltage signal. A considerable reduction of $93 \%$ of the displacement r.m.s. values of the tool tip, was obtained when simulating this control system.
\end{abstract}

Key words: Tool vibration, active control, self-sensing actuator, turning process

\section{INTRODUCTION}

In machining, and particularly in turning processes, tool vibration is a problem that commonly has to be dealt with, mainly because of its constraining effect on productivity, workpiece surface finish and tool life. This vibration constrains productivity since the selection of cutting parameters is restricted to values lying in a region in which they guarantee a stable but not the most productive operation (Chen et al., 1993; Yeh and Lai, 1995). Tool vibration affects the surface finish of work pieces during cutting owing to the relative motion of the tool and the work piece (Cheung and Lee, 2000). Compression and friction during chatter are additional sources of energy dissipation, which promote flank wear on the cutting tool and reduce tool life (Chiou and Liang, 2000).

Control over tool vibration can be approached, by using passive methods in which either structural stiffness is added to the lathe or a tuned vibration absorber is applied to the 
process (Tarng et al., 2000; Lee et al., 2001). However, active control has become a favored method since significant advances have been made in the development of micro-positioning actuators over the past decade. These developments allowed a choice between semi-active and fully active vibration absorbers. Semi-active means that the control force is located between the primary and absorber structure, whereas active means that the control force is applied more directly, between ground and the primary structure directly (Pratt and Nayfeh, 1997). The type of actuator considered in this work is a piezoelectric one. An earlier example of active tool vibration control, in turning processes, which also made use of a piezoelectric actuator is represented by Choudhury et al. (1997). Examples of active tool vibration control are represented by the work done by Andrén et al. (2003) and Pan and Su (2001). They respectively used adaptive filter and adaptive control methods to handle process uncertainties.

In the control of tool vibrations, the problems other than process uncertainties are the lack of space around the tool tip and the additional cost of a sensor to pick up the tool displacements. This work shows through simulation that these two problems can be solved when a self-sensing actuator (SSA) is employed in the control system. Piezoceramics can be used in this fashion, because it is possible to exploit the piezoelectric and reciprocal effect simultaneously. Besides the piezoelectric effect of piezoceramics, i.e. the generation of a voltage if a force load is applied to such a material, it also exhibits a reciprocal effect, which consists of undergoing a change in length when a voltage is applied across its electrodes. This means that the piezoactuator can simultaneously function as a sensor, i.e. as a SSA. The possibility of functioning as an SSA has already been investigated by Dosch and Inman (1992) and was then covered in further detail by Anderson and Hagood (1994). In turning processes, this principle can be used as a SSA in a closed-loop tool vibration control system. Therefore, by using an SSA instead of a separate actuator and sensor, the abovementioned problems can be overcome.

This work addresses critical issues in the simulation of tool vibration control in turning processes, using a SSA. The paper answers the main question of how the displacement of the tool tip, as a result of both the cutting force and the actuator force, can be measured when the SSA is placed at an offset from the tool tip. In this case, one could anticipate that models representing the force displacement relationships between the tool tip and the actuator and vice versa, would play a significant role in the simulation. The paper therefore shows how the actuator structure of the passive tool holder as well as the sensing path of the SSA may be modeled. It furthermore describes how these models, in the form of IIR-filters, can be incorporated into the feedback-filtered-x least-mean-square (LMS) adaptive algorithm. The stability problems associated with the IIR-filter are dealt with by using the principle of prefiltering the error signal with the poles of the passive tool-holder structure (Vipperman et al., 1993). Another critical question, that this paper addresses, is what signal should be used to represent the cutting force during the simulation. Pertaining to this question this work gives details of how a representative cutting-force signal is obtained from a practical turning process and then processed for use in the simulation of tool vibration control. The results of a simulation of the control system give an indication as to what extent the attenuation of tool vibration is possible. 


\section{SELF-SENSING ACTUATOR (SSA) IN TURNING}

\subsection{Requirements to Consider}

Three equally important aspects need attention for the simulation of tool vibration control. They are firstly the question of a representative cutting-force signal, secondly the decision about a suitable actuator, and thirdly the requirement of an effective control method.

As this work deals with the simulation stage of vibration control, a signal was needed which could represent a typical cutting force in the turning process. To meet this need, the cutting force of a practical turning process was measured and the signal stored. This signal constitutes the source of vibration during simulation.

The decision about which actuator would comply with the requirements of the application under consideration was mainly based on the criterion that it should be self-sensing. The reasons for this criterion are the lack of mounting space at the tool tip and the additional cost of an otherwise separate sensor. Another requirement emanated from the nature of the turning process, which requires actuators that can control the vibration from high cutting forces at a high frequency. All these requirements for an actuator could be met by a piezoelectric type of actuator, which would satisfy the main need of serving simultaneously as an actuator and a sensor. The first purpose of the sensor is to pick up the resultant displacement of the tool tip, which can be used as feedback in a closed-loop control system. As the SSA is a collocated sensor-actuator pair situated at an offset from the tool tip (Figure 1), it will not be able to capture the true displacement of the tool tip. It will only sense a voltage, which is also influenced by the dynamics of the passive tool-holder structure. Therefore mathematical models of the passive tool-holder structure will be involved in the simulation of the control system. The position for the actuator at an offset from the tool tip requires the structural modes participating in the direction of the cutting force to be included in these models. Their digital representations should therefore be in the form of IIR-filters. These models are the elements of a dynamic flexibility matrix, which relates forces at two points of interest to their respective displacements. The two mentioned points of interest are the tool tip and the connecting point of the actuator and tool-holder. These are the points where internal and external forces respectively act in and onto the passive structure. The need for mathematical models can be satisfied numerically by generating a finite element model of the passive tool-holder structure in MSC-Patran and running a modal analysis in MSC-Nastran.

The third of the abovementioned aspects is the requirement for an active control method. It is most important that such a method should have the property of being adaptive, owing to the random and non-stationary nature of a turning process. A control algorithm often recommended in the literature on active vibration control because of its simplicity, is the filtered-x LMS (Fuller et al., 1996). Because this algorithm makes use of an adaptive filter it is suited to dealing with the abovementioned type of tool vibrations induced by the cutting force (Andrén et al., 2003). For the use of this control method, two problems have to be addressed. The first one is directly linked to the choice of the filtered-x LMS control algorithm. The use of infinite impulse response (IIR)-filters for the models, which form the elements of the dynamic flexibility matrix of the passive structure, introduce stability problems in an adaptive control algorithm such as the chosen LMS-type. This problem will therefore require stabiliz- 


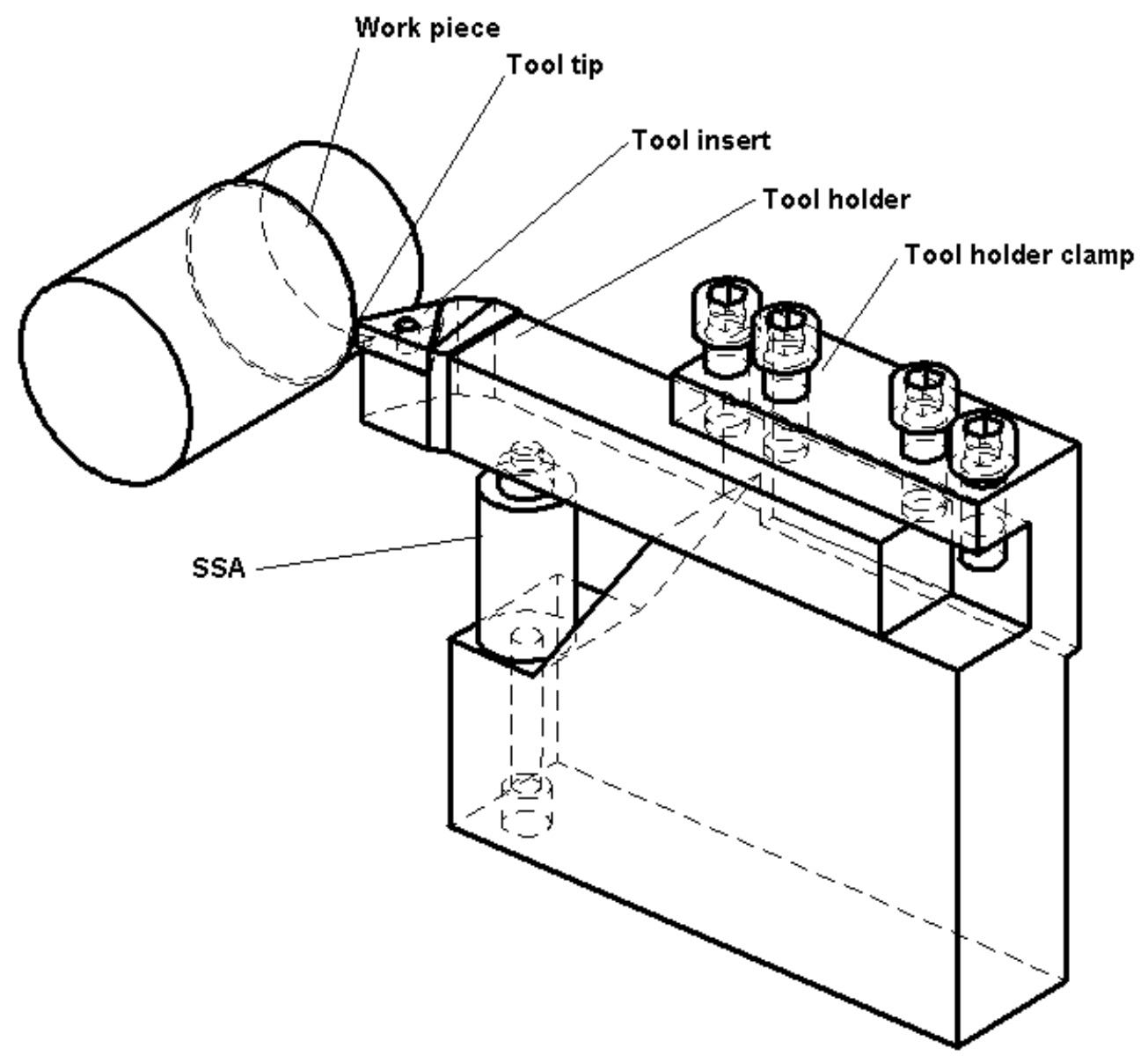

Figure 1. Tool-holder clamp with tool holder, self-sensing actuator (SSA) and work piece.

ing measures in the control system. The second problem, also linked to the LMS-type algorithm, is that a reference signal should be available to the adaptive filter and its algorithm. In the application to tool vibration control, the tool tip displacement, due to the control action and cutting forces should serve as a reference signal, but it cannot be measured. In relation to the first of these two problems, Vipperman et al. (1993) propose a technique, which is mentioned in the previous section and is used to avoid the stability problem. It involves the pre-filtering of the error signal, which is the tool tip displacement resulting from both the actuator and the cutting force input into the tool holder. The second problem is overcome by using a feedback version of the filtered-x LMS algorithm as proposed by Kuo and Morgan (1996), so that the need for a reference signal is met. This is possible because the cutting force measured for simulation purposes is close to being a narrowband disturbance. 


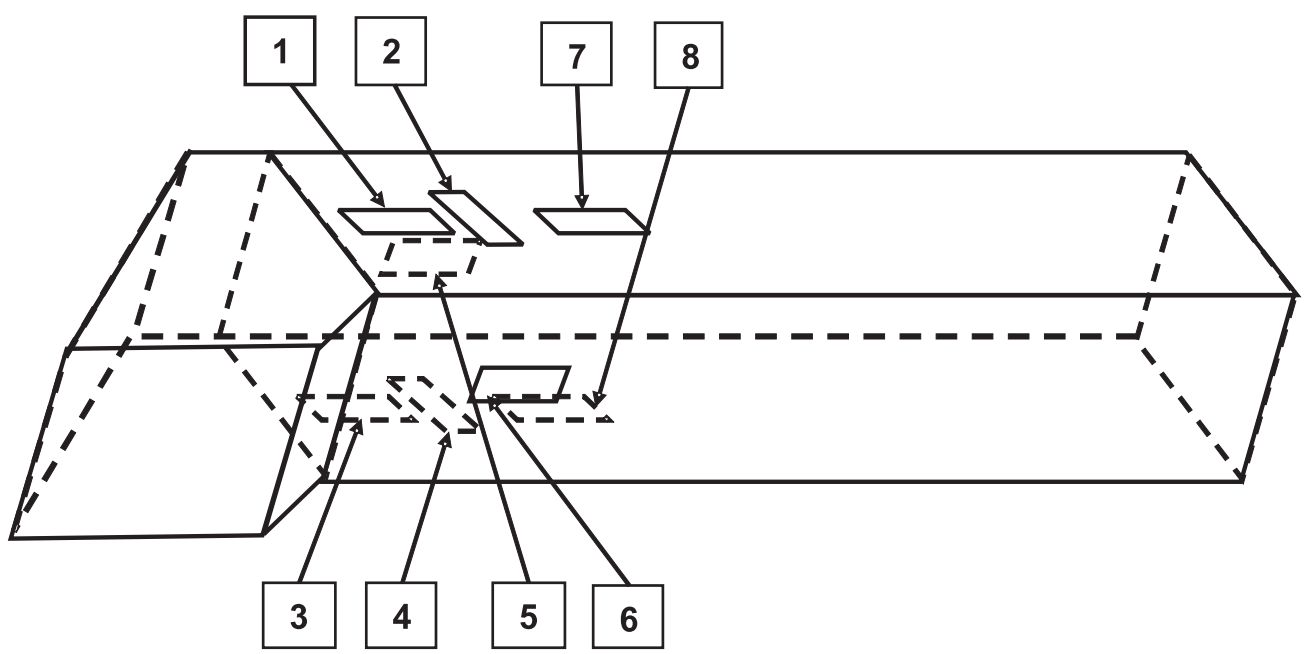

Figure 2. Tool holder with strain gauges (SG) .SGs 1 to 4 in full-bridge measure the thrust force; SGs 5 and 6 in half-bridge measure the feed force; SGs 7 and 8 in half-bridge measure the cutting force.

\subsection{Assumptions}

This work represents a preliminary investigation for the implementation of SSAs in turning. Various assumptions were made of which the validity will become clearer during future practical implementation. These assumptions are however believed to be reasonable.

- The cutting process $(\mathrm{CP})$ causes a cutting force at the tool tip and there is no feedback from the controlled tool tip to the $\mathrm{CP}$, i.e. the control system has no influence on the cutting force.

- The tool holder acts like a cantilever, which is supported by the SSA at an offset from the tool tip.

- The structure is lightly damped and a proportional viscous damping model is sufficient.

- In the envisaged implementation stage the sensing circuit will be of a full-bridge type. Here, the bridge is assumed to be balanced, i.e. the branch of the bridge parallel to the branch containing the SSA, contains an impedance component equal to that of the SSA.

\section{REPRESENTATIVE CUTTING-FORCE VIBRATION SOURCE}

Strain gauges mounted on a turning tool holder (Figure 2) were connected in bridge circuits and served as transducers to measure the cutting force. The signal data acquired were then post-processed so that they could be used as a typical vibration source in the simulation of the control system.

The cutting was done, by using a Mitsubishi TNMG 160408 UTi20T uncoated-carbide tool insert on EN 19 steel with the following cutting parameters: 


$\begin{array}{ll}\text { Depth of cut: } & 1 \mathrm{~mm} \\ \text { Feed: } & 0.3 \mathrm{~mm} / \mathrm{rev} \\ \text { Cutting speed: } & 1.40 \text { to } 2.07 \mathrm{~m} / \mathrm{s}\end{array}$

The cutting parameters were chosen from a region recommended by the insert manufacturer for that specific insert and work-piece material. However, the parameters were selected as low as possible to avoid being forced by reducing the work-piece diameter to make large changes in the speed on the lathe. The cutting speed was then chosen so that the total time of cutting-force measurement was kept as short as possible and corresponded to an insert life span of 95 minutes of cutting time.

The static cutting force was calibrated on the tool holder by means of mounted strain gauge circuits. The cutting force was thus assumed to be a result of the tool holder's displacement only and the influence of the tool holder's inertia on this force was neglected, which is believed to be a conservative approach. The calibration was performed by measuring voltages, $v_{i}$, while applying three different known forces in the $j$ th direction and zero force in the remaining directions.

Using the three different force/voltage pairs, each coefficient $a_{i j}$ in the $j$ th column of the calibration matrix A was determined three times from the relationship defined as

$$
\mathbf{v}=\mathbf{A} \mathbf{f}_{c}
$$

where the force vector $\mathbf{f}_{c}=\left[\begin{array}{lll}f_{c x} & f_{c y} & f_{c z}\end{array}\right]^{T}$ and the average were then taken for each coefficient. The force vector signals, $\mathbf{f}_{k}$, could then be obtained from the measured voltage signals $\mathbf{v}_{k}=\left[\begin{array}{lll}\mathbf{v}_{1 k} & \mathbf{v}_{2 k} & \mathbf{v}_{3 k}\end{array}\right]^{T}$ with

$$
\mathbf{f}_{k}=\mathbf{A}^{-1} \mathbf{v}_{k},
$$

where the subscript $k$ refers to the signal sequence at time $t_{k}$ and will henceforth be used as such. Furthermore, only the force contributions in the direction of the cutting force were used in the simulation.

Post-processing of the calibrated signal was done, by removing the drift and noise contribution of the amplifier in the measurement line by passing the signal through zero-phasedistortion high-pass and notch filters. The post-processed force signal can be used for simulation purposes. An IIR-filter representing the modal model of the passive tool holder was applied to the cutting force signal that was obtained after calibration. This filtering is done in order to deliver displacement values. The abovementioned filter and force signal together constitute the vibration source used for the simulation of control over the tool vibration of a turning process.

In this work, two tool-holder structures are considered. One is the tool holder equipped with strain gauges (as in Figure 2) and not supported by an actuator, and the other is the tool holder supported by an actuator. The first one is the tool holder used for the measurement of the cutting force. The second tool holder is the passive structure used as a model in the simulation of the vibration control system. The tool holder which was used for cutting-force measurements has its modes at frequencies differing from those of the passive tool holder shown in Figure 1, because the tool holder used for measuring the cutting force was not supported by an actuator whereas an actuator did support the passive tool holder used for 


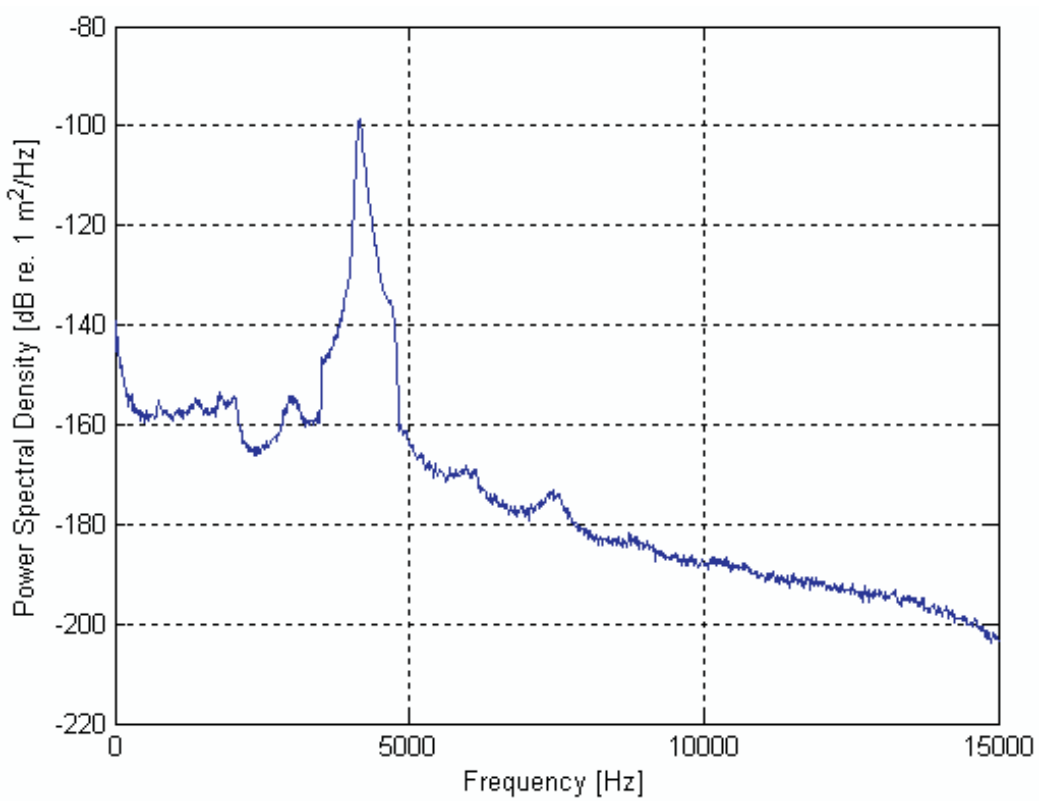

Figure 3. Power spectral density of the tool-tip displacement signal due to cutting force.

the simulation. Moreover, the additional mass of the strain gauges, strain gauge protection and cabling influenced the dynamics of the tool holder used for the cutting-force measurement. The tool holder only passes those frequency components of the cutting force, which are near the frequencies of the tool holder's modes participating in the cutting force direction. Therefore the tool holder acts as a mechanical filter. The measured signal is the one that was passed by the tool holder used for the cutting-force measurement, which has modes at lower frequencies than the equivalent modes of the passive tool-holder model used for simulation purposes. Therefore the measured cutting-force signal had frequency components corresponding to the frequency of the participating mode of the tool holder used for its measurement. These frequency components were shifted to the frequency range of the equivalent mode of the passive tool-holder model in order to obtain a new, adapted cutting-force signal.

The adapted cutting-force signal mentioned above was passed through an IIR-filter representation of $T_{11}(s)$, as mentioned before, which is the transfer function between the cutting force, $f_{1}(t)$, exerted on the tool tip and the displacement of the tool tip (see Figure 1) due to the cutting force. This filtering produced a displacement signal, $d(t)$, which is the displacement contribution of the cutting force to the total displacement of the tool tip. The power spectral density of this signal is shown in Figure 3. In this vibration-control approach signal, $d(t)$, is regarded as narrowband; a feedback version of the filtered-x LMS-algorithm could therefore be used for tool-tip vibration control. Figure 4 shows that the probability distribution of the signal, $f_{1}(t)$, is a Gaussian process for the first 367500 samples but indicates a nonlinearity during the two passes thereafter. This process nonlinearity was observed to occur during different passes on three different lathes. No precise explanation of this phenomenon has as yet been found. It may be due to an interaction between the cutting parameters chosen and radial play on the spindle bearings. 


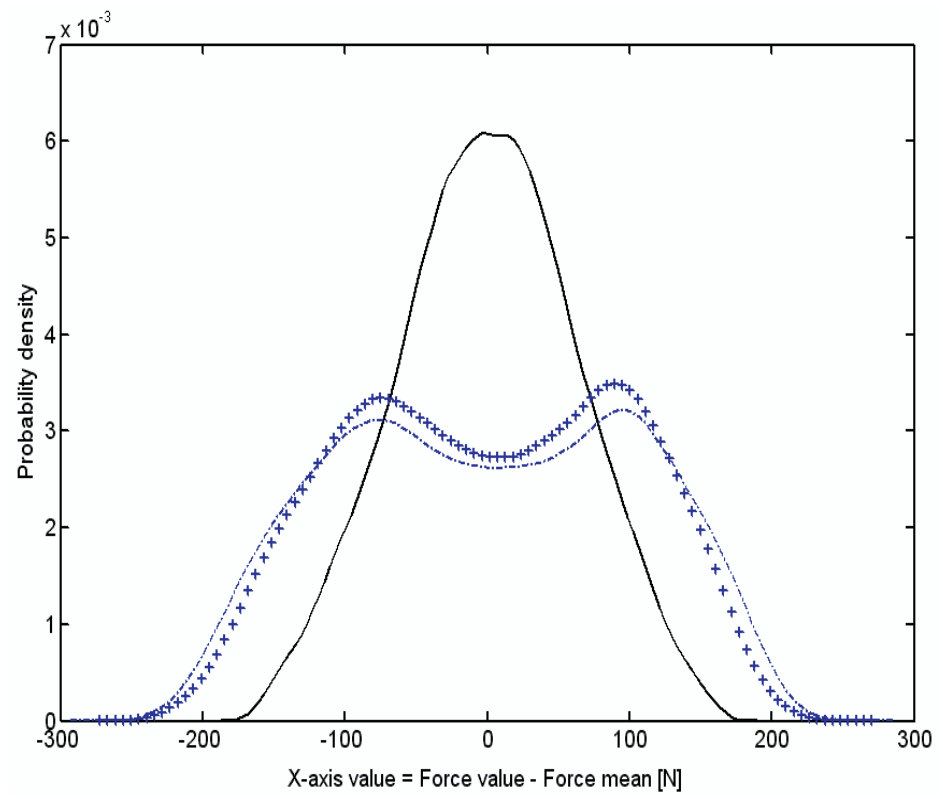

Figure 4. Probability density estimate of cutting-force signal. First pass (solid line), second pass (dotted line), third pass (crossed line) - each pass is equivalent to 367500 samples at $F_{s}=15000 \mathrm{~Hz}$.

\section{MODELING OF THE PASSIVE TOOL HOLDER}

Modeling is here started in the s-domain because literature most commonly deals with modeling theory for the control of structures in this domain.

The tool-holder structure and the inactive SSA, positioned as shown in Figure 1, constitute a passive structure. The vertical tool-tip displacement is here called DOF 1 (first degreeof-freedom) and the vertical displacement of the actuator tool-holder connecting point is called DOF 2 (second degree-of-freedom) as shown in Figure 5. DOF 2 was introduced because the SSA senses only the displacement of DOF 2, from which the displacement which here needs to be controlled, i.e. DOF 1, can be constructed mathematically. The shown passive structure has several modes. A constant or a finite impulse response (FIR) filter with only a few coefficients is therefore not sufficient to model the transfer behaviour from forces as inputs to DOF 1 and DOF 2 as outputs.

Modal models are used here to model the passive tool-holder structure and incorporate its modes. The first model, $T_{11}(s)$, also mentioned in the previous section, is a model representing the contribution of the cutting force at the tool tip, $f_{1}(t)$, to the displacement that would occur at the tool tip, $r_{1}(t)$. The second model represents the contribution of the force at the actuator structure's connecting point, $f_{2}(t)$, to the displacement, $r_{1}(t)$, at the tool tip and is denoted as $T_{12}(s)$. The third model is $T_{22}(s)$. It is part of the term in equation (4) which is the contribution of the force, $f_{2}(t)$, at the connecting point to the displacement of the connecting point, $r_{2}(t)$. Specifically in this work, which is a simulation using a selfsensing actuator, $T_{22}(s)$ serves as a model in the contribution of $f_{2}(t)$ to the displacement, 


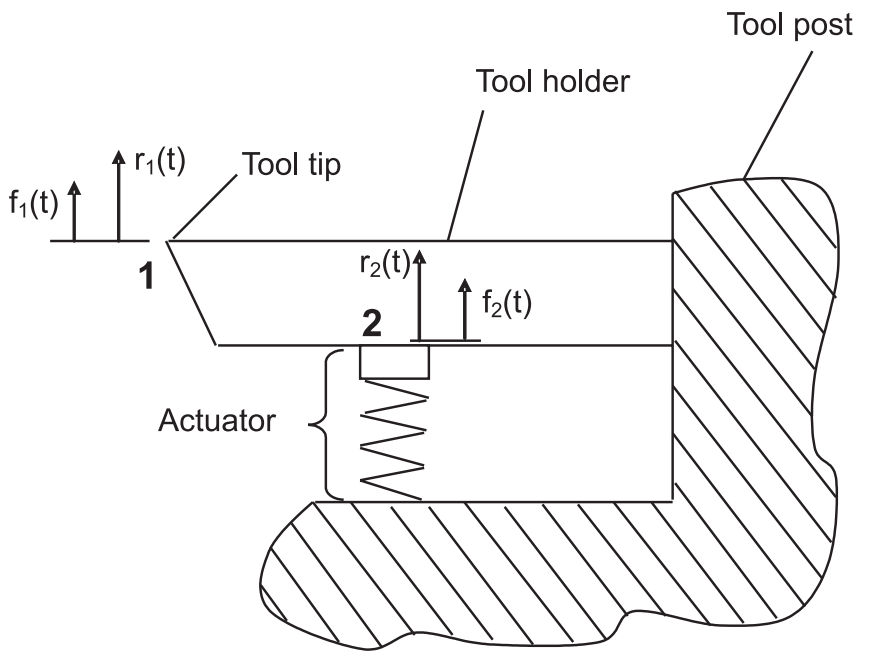

Figure 5. Tool holder and actuator with cutting and actuator forces.

$r_{2}(t)$, which is equivalent to what the SSA measures, as in equation (4). The displacements at each DOF, $r_{1}(t)$ and $r_{2}(t)$, is caused by the joint influence of the two forces $f_{1}(t)$ and $f_{2}(t)$ at these two points. So two equations are necessary to model the displacements of DOF 1 and DOF 2 because of the forces at these two points. They are given as follows in the Laplace-domain:

$$
\begin{aligned}
& R_{1}=T_{11}(s) F_{1}+T_{12}(s) F_{2} \\
& R_{2}=T_{21}(s) F_{1}+T_{22}(s) F_{2},
\end{aligned}
$$

and because of symmetry, $T_{21}(s)=T_{12}(s)$. Three modal models are therefore needed to model the passive tool-holder structure and incorporate its modes.

In the frequency domain, the relationship between displacement vector $\mathbf{R}$ and input force vector $\mathbf{F}$, may be described by the dynamic flexibility matrix as

$$
\mathbf{R}=\left[-\omega^{2} \mathbf{M}+j \omega \mathbf{C}+\mathbf{K}\right]^{-1} \mathbf{F}=\mathbf{T}(\omega) \mathbf{F}
$$

and with $\boldsymbol{\Phi}=\left[\bar{\phi}_{1}, \bar{\phi}_{2} \ldots \bar{\phi}_{N}\right]$, where $\bar{\phi}_{i}$ is the orthonormal modal vector of the $i$ th mode, using modal coordinates

$$
\mathbf{R}=\boldsymbol{\Phi} \mathbf{Z}=\boldsymbol{\Phi} \operatorname{diag}\left\{\frac{1}{\left(\omega_{i}^{2}-\omega^{2}+2 j \xi_{i} \omega_{i} \omega\right)}\right\} \boldsymbol{\Phi}^{T} \mathbf{F},
$$

where $\mathbf{Z}$ is a vector of modal coordinates and where $\omega_{i}$ is the natural frequency, and the constant $\xi_{i}$ represents the viscous modal damping ratio of mode $i$ respectively. A modal damping ratio of $\xi_{i}=0.01$ (Preumont, 2002) has been used throughout. The frequency responses $T_{11}(j \omega), T_{12}(j \omega)$ and $T_{22}(j \omega)$ are elements of the dynamic flexibility matrix, 


$$
\mathbf{T}(\omega)=\sum_{i=1}^{N} \frac{\bar{\phi}_{i} \bar{\phi}_{i}^{T}}{\left(\omega_{i}^{2}-\omega^{2}+2 j \xi_{i} \omega_{i} \omega\right)} .
$$

These modes were obtained from an eigenvalue extraction by means of the Lanczos method using MSC Nastran.

The passive tool holder structure was modeled in MSC Patran as a cantilever supported by the SSA at an offset from the tool tip similarly to the configuration in Figure 5 . The PTJNR-2020-16A tool holder was modeled with 6312 hex-8 elements in a finite element method (FEM), such that the $20 \times 20 \mathrm{~mm}$ cross section consisted of $10 \times 10$ elements. Those nodes corresponding to the points of contact when clamped in the tool post of a Colchester Student lathe with $69.7 \mathrm{~mm}$ overhang were constrained in all translational and rotational directions. In order to account for the SSA connected to the tool holder, a grounded spring and a point mass were modeled and added at the SSA's point of connection to the tool holder. With respect to this point of connection the overhang of the tool holder is $31.4 \mathrm{~mm}$. The point mass was obtained from

$$
m=\frac{K_{a}}{(2 \pi f)^{2}}
$$

where $K_{a}$ and $f$ are the stiffness of $120 \mathrm{kN} / \mathrm{mm}$ and resonance frequency of $27 \mathrm{kHz}$, respectively, specified for the SSA. The values for this point mass and spring stiffness were those specified in technical data for the Pst 500/10/15 VS 18 actuator (from Piezomechanik $\mathrm{GmbH})$.

The clamping position of the tool holder as shown in Figure 1 is equivalent to the position when the cutting force was measured with the strain-gauged tool holder as described in the previous section. This position was at an increased distance from the tool tip to provide space for strain gauges. For the purposes of vibration control simulation, the same clamping position is used. However, the simulations are based on tool-tip displacement sensing that is done not by means of strain gauges but by means of a SSA instead.

Figures 6 to 8 show the FEM-calculated frequency response functions of the three models. Whereas the frequency responses in Figures 6 and 8 are typical for collocated inputoutput pairs, the frequency response shown in Figure 7 is typical of a non-collocated inputoutput relationship because it is not showing an anti-resonance behind the second mode resonance. It can be seen that the magnitudes of the frequency responses at the resonant frequencies correspond to the residual values in Table 1.

The residuals, $\phi_{i_{j}} \phi_{i_{j}}$, of the three modal models are given in Table 1 with the subscript, $j$, referring to the respective DOF. These graphs as well as the residuals, from Table 1, indicate that modes above the chosen participating mode, mode 2 , have magnitudes less significant than the magnitude of mode 2.

\section{MODELING OF THE ACTIVE TOOL HOLDER}

This section shows how the active actuator is incorporated into the structure with the relationship between the voltage applied to the actuator and the force generated by it, based on a derivation by Preumont (2002). 

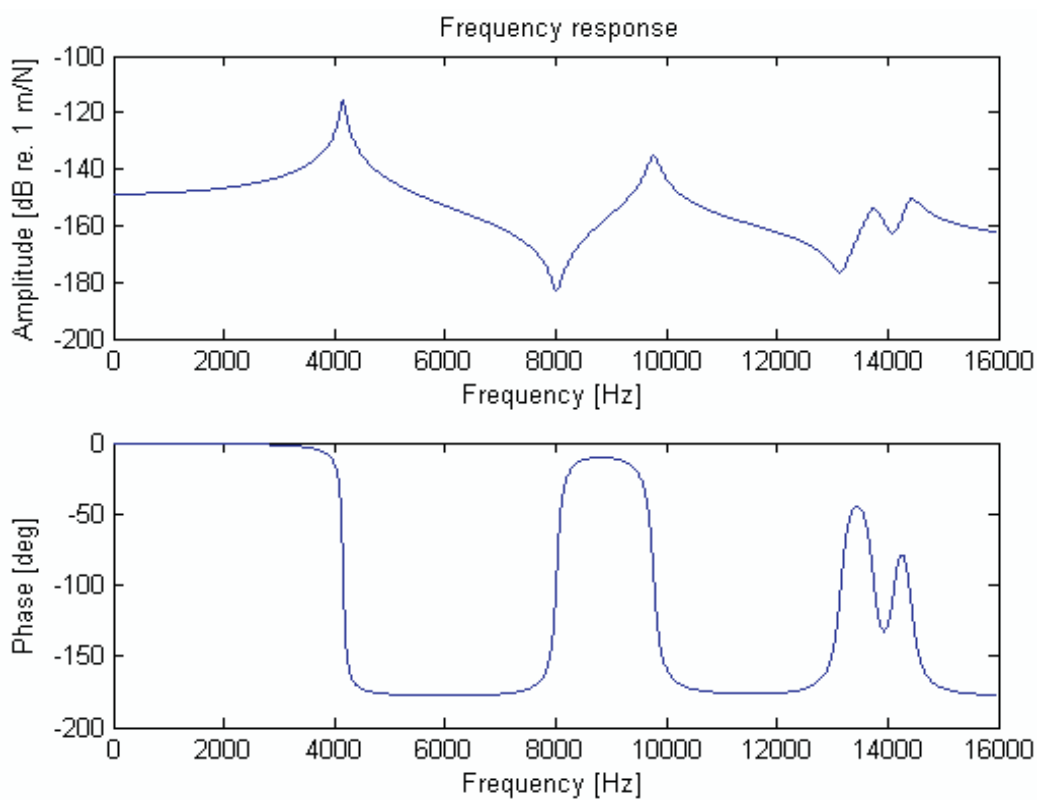

Figure 6. Frequency response of tool-holder collocated cutting-force path with cutting force as input and tool-tip displacement as output (first five participating modes of which the first is invisible).
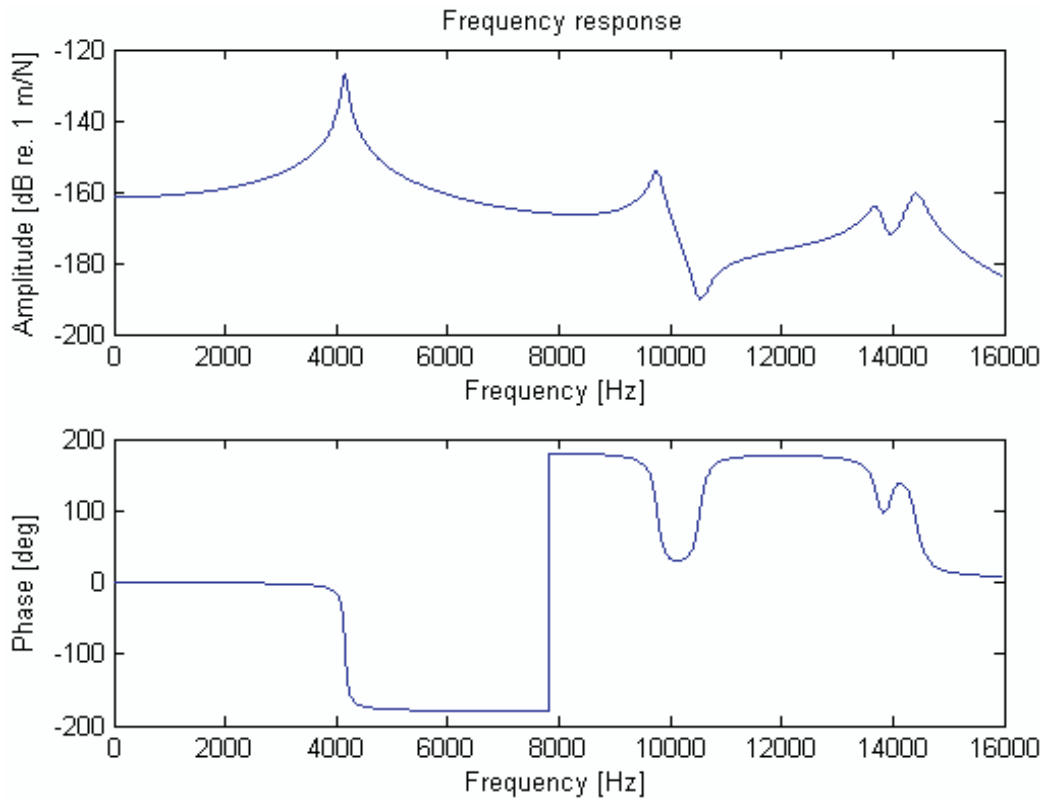

Figure 7. Frequency response of passive tool-holder forward path with actuator force as input and tool-tip displacement as output (first five participating modes, of which the first is invisible). 

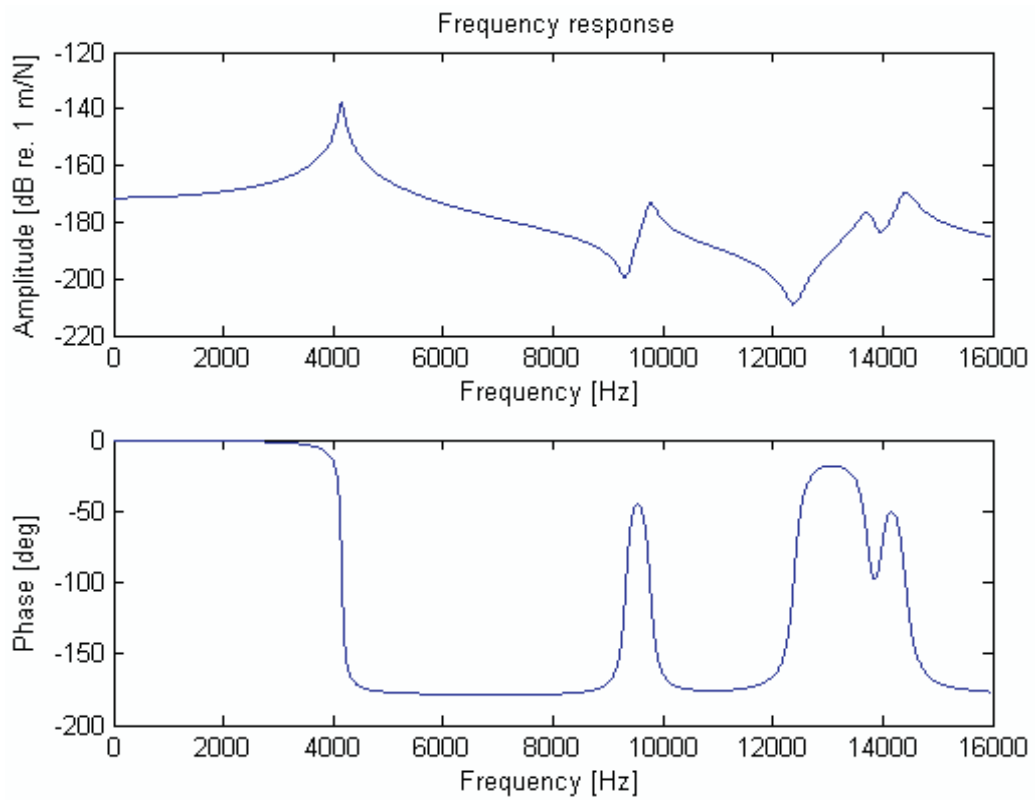

Figure 8. Frequency response of passive tool-holder collocated actuator path with actuator force as input and actuator extension as output (first five participating modes, of which the first is invisible).

Table 1. Natural frequencies and residual values of passive-structure models.

\begin{tabular}{lcccc}
\hline Mode $i$ & $\omega_{i} / 2 \pi[\mathrm{Hz}]$ & $\phi_{i_{1}} \phi_{i_{1}}$ of $T_{11}(\omega)$ & $\phi_{i_{1}} \phi_{i_{2}}$ of $T_{12}(\omega)$ & $\phi_{i_{2}} \phi_{i_{2}}$ of $T_{22}(\omega)$ \\
\hline 1 & 2859.6 & 0.0002 & 0.0002 & 0.0002 \\
2 & 4152.7 & 21.8427 & 6.1581 & 1.7361 \\
3 & 9764.6 & 12.8141 & -1.4122 & 0.1556 \\
4 & 13735 & 2.8190 & -0.7420 & 0.1953 \\
5 & 14412 & 4.3376 & -1.5224 & 0.5343 \\
\hline
\end{tabular}

If a voltage, $u$, is applied to the actuator, its unconstrained extension is

$$
\delta=g_{a} u,
$$

where $g_{a}$ is the gain of the actuator, $g_{a}=n d_{33}$, with $n$ the numbers of layers in the stack actuator and $d_{33}$ a piezoelectric constant in $[\mathrm{m} / \mathrm{V}]$.

The piezoelectric load along a line of action coaxial with the actuator is considered in the same fashion as if it were a thermal load. It is computed as

$$
f_{p}=K_{a} \delta
$$

where $K_{a}$ is the stiffness of the actuator. The actual load on the tool-holder structure at point $2, f_{e l_{2}}(t)$ will be proportional to the relative expansion, i.e. the elastic change in the 
length of the actuator. The elastic change in the length is the difference between the actual displacement of point 2, $r_{2}(t)$, and the unconstrained expansion, $\delta(t)$, for the given applied voltage, $u(t)$. Therefore $f_{e l_{2}}(t)$ is given as

$$
f_{e l_{2}}(t)=K_{a}\left(r_{2}(t)-\delta(t)\right) .
$$

If $f_{p}$ is handled in the same way as a thermal load, it can be made part of the stiffness force vector of the homogeneous equation of motion of the passive structure, so that the element of that vector corresponding to DOF 2 is equal to $f_{e l_{2}}(t)$. If the $f_{p}$ term of $f_{e l_{2}}(t)$ is then brought to the right-hand side of the initially homogeneous equation of motion for the passive structure, the equation of motion then becomes

$$
\mathbf{M r}+\mathbf{C} \dot{\mathbf{r}}+\mathbf{K r}=\mathbf{f}
$$

where $f_{p}$ is an element of the force vector $\mathbf{f}$ and $\mathbf{M}, \mathbf{C}$ and $\mathbf{K}$ are the mass, damping and stiffness matrices respectively of the passive structure, which in this way also incorporate the mass of the actuator and its stiffness $K_{a}$. When substituting $r_{2}(t)$ in equation (11) with modal coordinates, one obtains

$$
f_{e l_{2}}(t)=K_{a}\left(\sum_{i=1}^{N} z_{i}(t) \phi_{i_{2}}-g_{a} u\right) .
$$

From equation (6) one can see that the modal coordinates, $z_{i}$, in the frequency domain are

$$
\mathbf{Z}(\omega)=\operatorname{diag}\left\{\frac{1}{\left(\omega_{i}^{2}-\omega^{2}+2 j \xi_{i} \omega_{i} \omega\right)}\right\} \boldsymbol{\Phi}^{T} \mathbf{F}(\omega) .
$$

For the $i$ th mode, equation (14) becomes

$$
Z_{i}(\omega)=\frac{1}{\left(\omega_{i}^{2}-\omega^{2}+2 j \xi_{i} \omega_{i} \omega\right)} \sum_{l=1}^{N_{d o f}} \phi_{i_{l}} F_{l}(\omega),
$$

where $N_{d o f}$ is the number of degrees-of-freedom. Then for $F_{l}(\omega)=0 \forall l \neq p, Z_{i}(\omega)$ becomes

$$
Z_{i}(\omega)=\left(\frac{\phi_{i_{p}}}{\omega_{i}^{2}-\omega^{2}+2 j \xi_{i} \omega_{i} \omega}\right) F_{p}(\omega) .
$$

In order to derive a relationship between the applied actuator voltage, $U(\omega)$, and the force, $F_{e l_{2}}(\omega)$, exerted by the actuator on the structure at point 2 , equation (13) becomes

$$
\frac{F_{e l_{2}}}{U}=K_{a}\left(\sum_{i=1}^{N} \frac{Z_{i} \phi_{i_{2}}}{U}-g_{a}\right) .
$$


Substituting the modal coordinates $Z_{i}$ of equation (16), and using $F_{p}(\omega)$ as the excitation at point 2 , equation (17) becomes

$$
\frac{F_{e l_{2}}}{U}=K_{a}\left(\sum_{i=1}^{N} \frac{\phi_{i_{2}} \phi_{i_{2}} F_{p}}{\left(\omega_{i}^{2}-\omega^{2}+2 j \xi_{i} \omega_{i} \omega\right) U}-g_{a}\right) .
$$

Replacing $U$ on the right-hand side of equation (18),

$$
\frac{F_{e l_{2}}}{U}=K_{a}\left(\sum_{i=1}^{N} \frac{\phi_{i_{2}}^{2} F_{p} K_{a} g_{a}}{\left(\omega_{i}^{2}-\omega^{2}+2 j \xi_{i} \omega_{i} \omega\right) F_{p}}-g_{a}\right),
$$

and simplifying equation (19), the transfer function from $U(\omega)$ to $F_{e l_{2}}(\omega)$ becomes

$$
\begin{aligned}
\frac{F_{e l_{2}}}{U} & =K_{a} g_{a}\left(\sum_{i=1}^{N} \frac{\phi_{i_{2}}^{2}}{\left(\omega_{i}^{2}-\omega^{2}+2 j \xi_{i} \omega_{i} \omega\right)} K_{a}-1\right) \\
& =K_{a} g_{a}\left(T_{22}(\omega)-1\right)=K_{a} g_{a} T_{a}(\omega),
\end{aligned}
$$

where $F_{2}=F_{e l_{2}}$.

\section{THE CONTROL METHOD}

By means of the zero-order-hold all the s-domain transfer functions, $T_{11}(s), T_{12}(s), T_{22}(s)$ and $T_{a}(s)$ were converted to discrete filters in the z-domain and subsequently used as such $T_{11}(s), T_{12}(s), T_{22}(s)$ and $T_{a}(s)$ are proper transfer functions of which inversions are not realizable. But because the discrete versions of these models and inversions of some of them had to be used as part of the control algorithm they were inverted by incorporating their numerators in an all-pole IIR-filter and their denominators in an all-zero FIR-filter.

In this section, lower-case bold symbols are used for the vectors that represent sample sequences. All the subscripts $k$ in this section indicate a sample sequence of a signal at time $t_{k}$.

Proceeding with the model of the previous section, i.e. the tool holder supported by the passive actuator, Figure 9 shows how the transfer behavior of the passive structure is accounted for in the simulation of the control system. That part of the block diagram surrounded by the dashed line constitutes the plant and the SSA, as represented during simulation. In the simulation used in this work, the plant and the SSA, are represented by filters as shown in Figure 9. In this control system the plant is simulated with the path from the voltage, $\mathbf{u}_{k}$, applied as a control input into the actuator and the tool-tip displacement, $\mathbf{y}_{k}$, induced by the actuator as the plant output. The voltage, $\mathbf{v}_{s k}$, is the voltage sensed by the SSA as a result of the displacement along DOF 2. The tool-tip displacement due to the cutting force, $\mathbf{f}_{1 k}$, is represented here by $\mathbf{d}_{k}$ and the resulting tool-tip displacement is denoted by $\mathbf{e}_{k}=\mathbf{r}_{1 k}$. The simulation of the voltage sensed by the SSA, $\mathbf{v}_{s k}$, due to the total displacement of the tool tip, $\mathbf{e}_{k}$, i.e. the sensing path, is that part of the block diagram lying between $\mathbf{e}_{k}$ and $\mathbf{v}_{s k}$. 


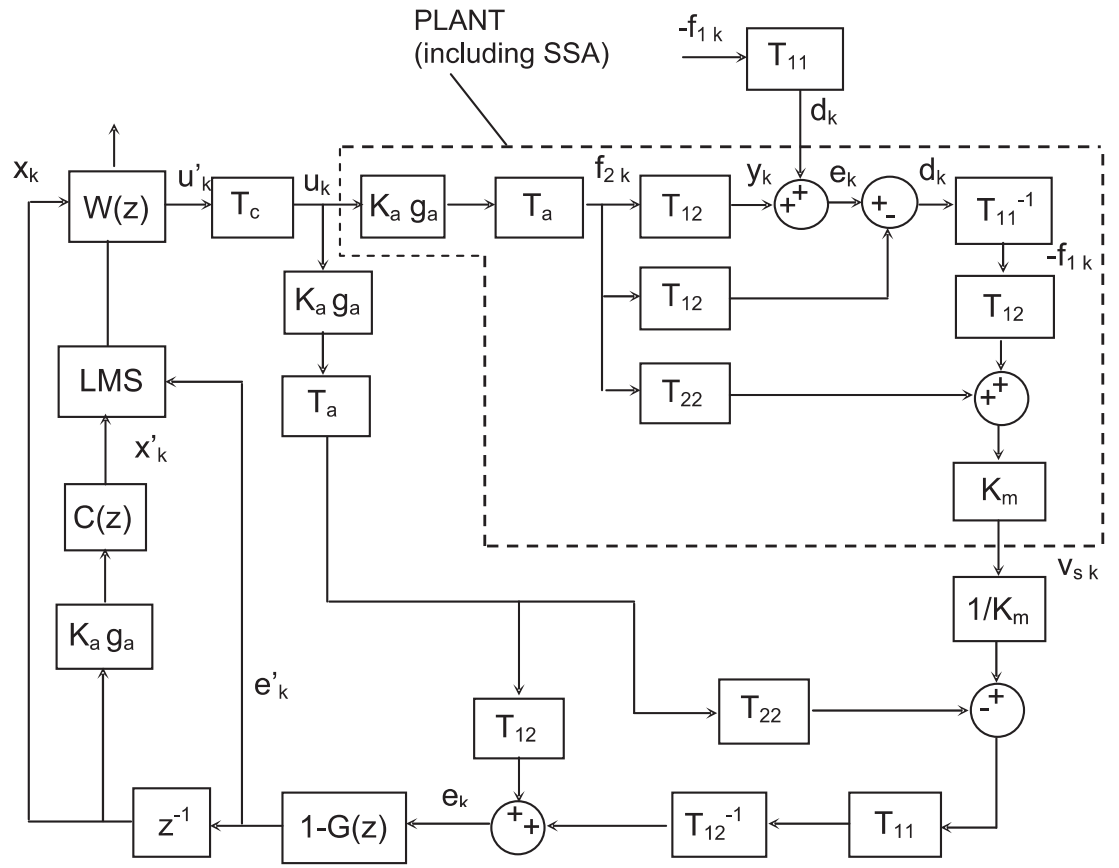

Figure 9. Block diagram of the tool vibration control system.

$T_{a}(z)$ in the forward path, i.e. the plant, induces a behavior which is initially divergent and finally convergent in its step response. Since the poles and zeros of $T_{a}(z)$ are located inside the unit-circle of the z-plane, one can apply pole-zero cancellation of $T_{a}(z)$ in the controller, which is that part in Figure 9 outside the dashed line. $T_{c}(z)=T_{a}^{-1}(z)$ is therefore used in the controller to compensate for $T_{a}(z)$ which induces the abovementioned divergent/convergent behavior.

$T_{12}(z)$ is a simulation of the plant since its input is the actuator force, $\mathbf{f}_{2 k}$, and the tool-tip displacement, $\mathbf{y}_{k}$, is its output. The displacement at point 2 (see Figure 5) is simulated by using equations (3) and (4) and solving for $\mathbf{r}_{2 k}$. This displacement is due to the forces to which the structure, including the structural effects of the actuator, is subjected during the turning process. The voltage sensed by the SSA, $\mathbf{v}_{s k}$, at point 2 is

$$
\mathbf{v}_{s k}=-Z_{m}(z) \Theta \mathbf{r}_{2 k}=K_{m} \mathbf{r}_{2 k}
$$

where $Z_{m}(z)$ represents the sensing circuit and $\boldsymbol{\Theta}$ describes the SSA parameters according to the notation used by Anderson et al. (1994). The latter two are furthermore accommodated in the constant gain, $K_{m}$, since all complex variables cancel out when the impedances appearing in the sensing circuit are assumed to consist of capacitors only.

Operations exactly opposite to those in the simulation of $\mathbf{r}_{2 k}$ are then done on $\mathbf{v}_{s k}$ in order to recover the error, $\mathbf{e}_{k}$, which is needed to compute the coefficients of the adaptive filter $W(z)$. This section describes how this computation is done. 
The model of the passive structure's forward path between a force input at the actuator position and its contribution to the total displacement of the tool tip as output, has poles and zeros and is given as

$$
T_{12}(z)=\frac{C(z)}{1-G(z)}=\frac{\sum_{i=0}^{L C-1} c_{i} z^{-i}}{\left(1-\sum_{j=1}^{L G} g_{j} z^{-j}\right)} .
$$

where $L C$ and $L G$ are filter lengths.

The transfer function of the passive structure with the force and the displacement at the actuator connection point as the collocated input and output respectively, has the same form as in equation (22) above, and is given as

$$
T_{22}(z)=\frac{P(z)}{1-B(z)}=\frac{\sum_{i=0}^{L P-1} p_{i} z^{-i}}{\left(1-\sum_{j=1}^{L B} b_{j} z^{-j}\right)} .
$$

where $L P$ and $L B$ are filter lengths again.

The error, $\mathbf{e}_{k}$, which is the resulting displacement of the tool tip in the direction of the cutting force, is given as

$$
\mathbf{e}_{k}=\mathbf{y}_{k}+\mathbf{d}_{k} .
$$

In order to obtain the plant output, $\mathbf{y}_{k}$, a force input from the actuator, $\mathbf{f}_{2 k}$, is given to the plant, $T_{12}(z)$. When replacing $\mathbf{y}_{k}$ in equation (24) by

$$
\mathbf{y}_{k}=T_{12}(z) \mathbf{f}_{2 k},
$$

$\mathbf{e}_{k}$ is obtained with a dependency on the control input, $\mathbf{u}_{k}^{\prime}$, as

$$
\mathbf{e}_{k}=K_{a} g_{a} T_{12}(z) \mathbf{u}_{k}^{\prime}+\mathbf{d}_{k} .
$$

This is the error, i.e. the resultant displacement of the tool tip. For the sake of simplicity in this section, it is assumed that $T_{c}(z)=T_{a}^{-1}(z)$ so that $T_{c}(z)$ and $T_{a}(z)$ are perfectly canceled. To prevent the poles of the passive tool-holder structure from participating in the error signal, the error, $\mathbf{e}_{k}$, is pre-filtered with the poles of $T_{12}(z)$. The new error, $\mathbf{e}_{k}^{\prime}$, becomes

$$
\mathbf{e}_{k}^{\prime}=(1-G(z)) \mathbf{e}_{k}=K_{a} g_{a} C(z) \mathbf{u}_{k}^{\prime}+(1-G(z)) \mathbf{d}_{k} .
$$

The minimization of $\mathbf{e}_{k}^{\prime}$ can be done by finding the coefficients, $w_{i}$, of the adaptive FIR-filter, that minimize the instantaneous squared error, expressed as the cost function

$$
J\left(w_{i}\right)=e_{k}^{\prime 2} .
$$


This search for coefficients, $w_{i}$, occurs along the direction of the negative gradient,

$$
\frac{\partial J}{\partial \mathbf{w}_{k}}=2 e_{k}^{\prime} \frac{\partial e_{k}^{\prime}}{\partial \mathbf{w}_{k}},
$$

towards the minima of the quadratic error surface, also known as performance surface. The coefficients, $w_{i}$, are updated for each time-step, using

$$
\mathbf{w}_{k+1}=\mathbf{w}_{k}-\mu \frac{\partial J}{\partial \mathbf{w}_{k}} .
$$

Since

$$
u_{k}^{\prime}=\sum_{i=0}^{L W-1} w_{i} x_{k-i},
$$

with $L W$ the adaptive filter length, the derivative term in the above equation is determined from

$$
\frac{\partial e_{k}^{\prime}}{\partial \mathbf{w}_{k}}=K_{a} g_{a} C(z) \mathbf{x}_{k},
$$

which is the reference signal, $\mathbf{x}_{k}$, filtered.

Inserting the corresponding terms, the final updating equation (30) for the adaptive filter coefficients becomes

$$
\mathbf{w}_{k+1}=\mathbf{w}_{k}-\alpha \mathbf{e}_{k}^{\prime} K_{a} g_{a} C(z) \mathbf{x}_{k},
$$

where $\alpha=2 \mu$ is a convergence coefficient, computed as in Elliott (2001) as

$$
\alpha=\frac{\tilde{\alpha}}{I \overline{\mathbf{x}_{k}^{\prime 2}}} .
$$

Here $\overline{\mathbf{x}_{k}^{\prime 2}}$ is the mean-square of the filtered reference signal, $\mathbf{x}_{k}^{\prime}$, as indicated in Figure 9, and $I$ represents the number of adaptive filter coefficients and

$$
0<\tilde{\alpha}<2
$$

One advantage of the LMS algorithm is that the terms in the cost function, such as the term due to the disturbance by the cutting force, which are not dependent on the adaptive filter weights, vanish in the gradient.

\section{SIMULATION RESULTS}

It was found that if the poles and zeros of the compensator, $T_{c}(z)$, are not accurately placed, i.e. $T_{c}(z) \approx T_{a}^{-1}(z)$, and their real and imaginary parts are $10 \%$ closer to the origin in the 
z-plane, an even better vibration control can be obtained. The simulations discussed in this section were therefore done under the abovementioned conditions.

Simulations were performed with the SSA in two different positions:

- Position A: The SSA is connected at the bottom surface of the tool holder at an offset from the tool-holder tip.

- Position B: The SSA is connected at the bottom surface at the tip of the tool holder.

Initially a sampling frequency of $F_{s}=45 \mathrm{kHz}$ was considered for the simulation of the vibration control system. With the SSA at position A, the percentage reduction of the displacement r.m.s. of the tool tip was only $88 \%$. It is interesting to note that in order to increase the performance of the vibration control by decreasing the frequency of the participating modes, a mass of approximately $0.57 \mathrm{~kg}$ would have to be added to the tool holder close to its tip. This would increase the overall reduction performance of the tool-tip vibration r.m.s. by $3 \%$. It is, however, more appropriate to increase the sampling frequency of the vibration control system to $F_{s}=90 \mathrm{kHz}$. The reason for choosing this sampling frequency is to enable the control system to function more accurately due to more accurate models. The improved accuracy results from a shorter one-sample delay which is introduced by the zero-order hold when the models are converted from the continuous s-domain to the discrete z-domain. The choice of the abovementioned two sampling frequencies was based on the commonly used guideline for the implementation of digital control systems, where $\omega_{s}=2 \pi F_{s}>10 \omega_{b}$. Here $\omega_{b}$ is the bandwidth of the closed-loop control system. In the control system, as used in this work, the bandwidth was taken to be equivalent to the highest participating mode, i.e. mode 2. Thus the lower bound for $\omega_{s}$ is $10 \omega_{b}$ but at $\omega_{s}=20 \omega_{b}$, $T(s) \approx T(z)$. This can be seen by inspection of the bode plots of $T(s)$ and $T(z)$ respectively. In Seborg et al. (1989) it can furthermore be seen that

$$
\lim _{T_{s} \rightarrow 0} H(z) T(z)=T(s),
$$

where $T_{s}$ is the sampling time and

$$
H(s)=\frac{1-e^{-s T_{s}}}{s}
$$

is the zero-order hold.

Figures 10 and 11 show the tool-tip displacements as the uncontrolled vibrations, $d(t)$, and the controlled vibrations, $e(t)$, in the form of a time domain plot for the actuator in positions A and B respectively.

Table 2 shows the percentage reductions of the displacement r.m.s. values for the resulting motion of the tool tip, $e(t)$, with the SSA in the abovementioned two positions. The results indicate that the percentage reduction of displacement r.m.s. with the SSA in position $\mathrm{A}$ is higher than with the actuator in position $\mathrm{B}$. When looking at the overall reduction, however, i.e. from the displacement r.m.s. with the passive SSA at position A to the displacement r.m.s. with the active SSA at position B, one can see that the reduction with the actuator in position $\mathrm{B}$ is higher than with the actuator in position $\mathrm{A}$. When the actuator is in position $\mathrm{B}$, therefore, the vibrations are reduced by a combination of the increased stiffness 

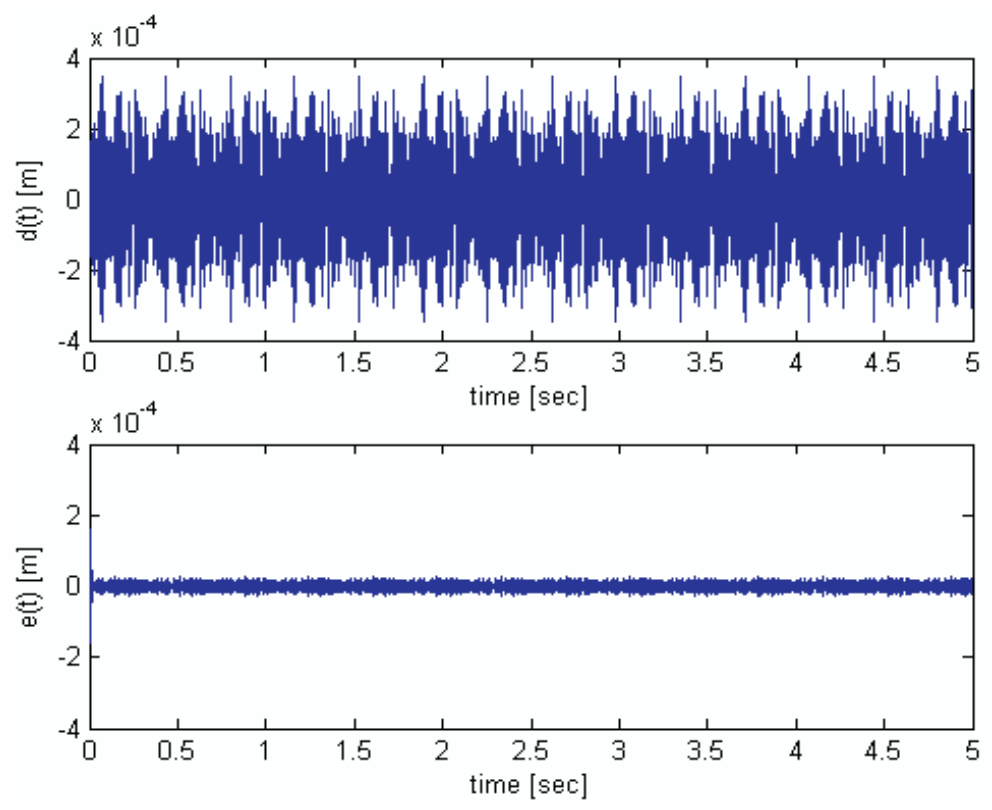

Figure 10. Cutting-force-induced vibrations, $d(t)$, and resultant vibrations, $e(t)$, with self-sensing actuator below the tool holder at an offset from the tool tip (position $\mathrm{A}$ ).
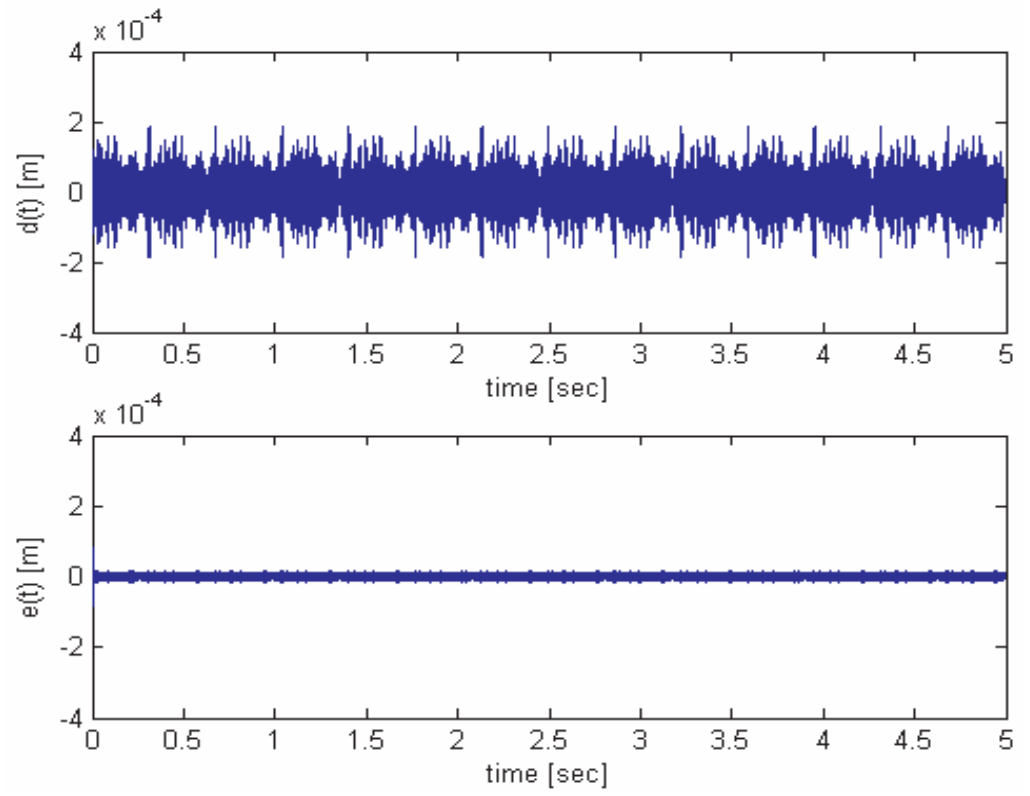

Figure 11. Cutting-force-induced vibrations, $d(t)$, and resultant vibrations, $e(t)$, with self-sensing actuator below the tool holder and tool tip (position $\mathrm{B}$ ). 
Table 2. Percentage reduction of displacement r.m.s., from $\mathbf{d}(t)$ to $\mathbf{e}(t)$, after 0.0111 secs.

\begin{tabular}{lccc}
\hline & $\mathbf{d}(\mathbf{t})[\mathrm{m}]$ & $\mathbf{e}(\mathbf{t})[\mathrm{m}]$ & Percentage reduction \\
\hline State of SSA & Passive & Active & \\
SSA at position A & $1.07 \times 10^{-4}$ & $7.52 \times 10^{-6}$ & $93 \%$ \\
SSA at position B & $5.29 \times 10^{-5}$ & $5.53 \times 10^{-6}$ & $90 \%$ \\
\hline
\end{tabular}

of the passive structure and the active vibration control. This shows that although having the SSA at position B is more effective in reducing the overall vibration, the SSA at position A would be a more practical way to control tool vibrations because it is out of the way of the tip where the cutting takes place.

Back to Figures 10 and 11 it is clear that they show the overall higher reduction in tool-tip displacement with the SSA passive at A to the displacement with the SSA active at B.

\section{LIMITATIONS}

Resulting from the structural model and the control system chosen, there are two main limitations to this concept.

- While the system is expected not to be limited to the cutting of specific work-piece shapes or materials, it is assumed that a specific tool post, tool holder and insert is used in a single-point turning process and that the clamping conditions are repeatable.

- The unique feature of this self-sensing concept compared to conventional control with direct measurement of the plant output, is that here the plant output has to be recovered from a signal picked up by the SSA. This is done by using models of the structure. The accuracy of the recovered plant output thus depends on the accuracy of the models. The results obtained in this simulation are expected to be the best possible results obtainable in practice because the models used for plant output recovery are identical to the models representing the plant.

\section{CONCLUSION}

It has been shown through simulation that the concept of a SSA can be applied to the turning process. IIR-filters were used in the simulation of the tool vibration control system to represent the discrete transfer functions of the passive tool-holder structure. The stability requirements for the use of IIR-filters in the mechanical structure of the control system had to be met by using the known principle of pre-filtering of the error signal with the poles of the structure. It has been shown that the position of the SSA plays a significant role in the performance of the control system and that by simulation a displacement r.m.s. reduction of 93\% is possible with the SSA in a practical position at an increased offset from the tool tip. 


\section{REFERENCES}

Anderson, E. H. and Hagood, N.W., 1994, "Simultaneous piezoelectric sensing/actuation: analysis and application to controlled structures," Journal of Sound and Vibration 174(5), 617-639.

Andrén, L., Håkansson, L., and Claesson, I., 2003, "Active control of machine tool vibrations in external turning operations," Proceedings of the Institution of Mechanical Engineers, Part B: Journal of Engineering Manufacture 217(6), 869-872.

Chen, S.-G., Ulsoy, A. G., and Koren, Y., 1993, "Computational stability analysis of chatter in turning," ASME Dynamic System Control Division Publications 50, 107-111.

Cheung, C. F. and Lee, W. B., 2000, "Theoretical and experimental investigation of surface roughness formation in ultra-precision diamond turning," International Journal of Machine Tools \&Manufacture 40(7), 979-1002.

Chiou, R. Y. and Liang, S. Y., 2000, "Analysis of acoustic emission in chatter vibration with tool wear effect in turning," International Journal of Machine Tools and Manufacture 40(7), 927-941.

Choudhury, S. K., Goudimenko, N. N., and Kudinov, V. A., 1997, "On-line control of machine tool vibration in turning," International Journal of Machine Tools and Manufacture 37(6), 801-811.

Dosch, J. J. and Inman, D. J., 1992, “A self-sensing piezoelectric actuator for collocated control," Journal of Intelligent Material Systems and Structures 3, 166-185.

Elliott, S. J., 2001, Signal Processing for Active Control, Academic Press Limited London.

Fuller, C. R., Elliott, S. J., and Nelson, P. A., 1996, Active Control of Vibration, Academic Press Limited, London.

Kuo, S. M. and Morgan, D. R., 1996, Active Noise Control Systems, John Wiley \& Sons, Inc, New York.

Lee, E. C., Nian, C. Y., and Tarng, Y. S., 2001, "Design of a dynamic vibration absorber against vibrations in turning operations," Journal of Materials Processing Technology 108(3), 278-285.

Pan, J. and Su, C.-Y., 2001, "Modeling and chatter suppression with ultra-precision in dynamic turning metal cutting process," in Proceedings of the ASME Design Engineering Technical Conference, Pittsburgh, PA, September 9-12, Vol. 6B, pp. 1125-1132.

Pratt, J. R. and Nayfeh, A. H., 1997, "Boring-bar chatter control using a two-axes active vibration absorber scheme," in Proceedings of the National Conference on Noise Control Engineering, Pennsylvania, PA, June 15-17, Vol. 2, pp. 313-324.

Preumont, A., 2002, Vibration Control of Active Structures, an Introduction, $2^{\text {nd }}$ edition, Kluwer Academic Publishers, Dordrecht, the Netherlands.

Seborg, D. E., Edgar, T. F., and Mellichamp, D. A., 1989, Process Dynamics and Control, Wiley, New York.

Tarng, Y. S., Kao, J. Y., and Lee, E. C., 2000, "Chatter suppression in turning operations with a tuned vibration absorber," Journal of Materials Processing Technology 105(1-2), 55-60.

Vipperman, J. S., Burdisso, R. A., and Fuller, C. R., 1993, "Active control of broadband structural vibration using the LMS algorithm," Journal of Sound and Vibration 166(2), 283-299

Yeh, L.-J. and Lai, G.-J., 1995, "Study of the monitoring and suppression system for turning slender work pieces," Proceedings of the Institution of Mechanical Engineers, Part B: Journal of Engineering Manufacture 209(B3), 227-236. 